\title{
Revisitando el concepto de la banalidad del mal desde la perspectiva del liderazgo de identidad
}

\section{Revisiting the concept of the banality of evil from the perspective of identity leadership}

\author{
Jesús M. Canto y Macarena Vallejo-Martín \\ Universidad de Málaga, España
}

\begin{abstract}
Resumen
El concepto de la banalidad del mal ha sido utilizado para explicar la maldad humana. Este concepto ha sido muy influyente en la psicología social y en el resto de las ciencias sociales. Los resultados obtenidos en los experimentos de Milgram sobre obediencia y en el experimento de la prisión de Stanford (EPS) han sido considerados como muestras de apoyo empírico al concepto de la banalidad del mal. Pero este concepto está recibiendo importantes críticas desde la historia y desde la propia psicología social. El reanálisis de los estudios de Milgram y del EPS no apoya las formulaciones teóricas clásicas formuladas por Milgram y Zimbardo. La variabilidad de los niveles de obediencia en los experimentos de Milgram y en el comportamiento mostrado por los guardias y prisioneros en el EPS requieren otro modelo teórico explicativo de tales resultados. Enmarcados dentro de la perspectiva de la identidad social, Haslam y Reicher proponen que el papel del liderazgo de identidad es un concepto clave para explicar los resultados obtenidos en estos estudios.
\end{abstract}

Palabras clave: agencia, obediencia, rol, liderazgo, identidad social

\begin{abstract}
The concept of the banality of evil has been used to explain human evil. This concept has been very influential in social psychology and in the other social sciences. Empirical support for this concept has been provided by the results obtained in the Milgram experiments on obedience and in the Stanford prison experiment (SPE), among others. However, it has received relevant criticism from the disciplines of history and social psychology. Reanalysis of the Milgram and EPE studies does not support the classic theoretical formulations offered by Milgram and Zimbardo. A different theoretical explanatory model of the results is needed in the light of variations in the levels of obedience in Milgram's experiments and in the behaviour of the guards and prisoners in the SPE. Framed within the perspective of social identity theory, Haslam and Reicher proposed that the role of identity leadership is a key concept to explain the results obtained in these studies.
\end{abstract}

Keywords: agency, obedience, role, leadership, social identity

Cómo citar este artículo: Canto, J. M., \& Vallejo-Martín, M. (2020) Revisitando el concepto de la banalidad del mal desde la perspectiva del liderazgo de identidad. Escritos de Psicología - Psychological Writings, 13(1), 34-45.

https://doi.org/10.24310/espsiescpsi.v13i1.10080

Correspondencia: Jesús María Canto Ortiz. Departamento de Psicología Social, Antropología Social, Trabajo Social y E.A.O. Facultad de Psicología y Logopedia. Universidad de Málaga. Blvr. Louis Pasteur, 25. 29010. MÁLAGA, España. E-mail: jcanto@uma.es. E-mail de la coautora Macarena Vallejo-Martín: mvallejo@uma.es. 


\section{Introducción}

Lewin (1951), uno de los psicólogos sociales más influyentes, planteó como postulado central de la teoría de campo que la conducta humana es función de la interacción entre la persona y el ambiente. Tal formulación se hacía eco de la importancia de los factores situacionales pero sin renunciar a los factores cognitivos (Álvaro y Garrido, 2003). Así la psicología social se situaba con Lewin en una perspectiva teórica que superaba las limitaciones de las tesis conductistas, lo que se constata en el hecho que el conductismo nunca fue una corriente predominante dentro de la psicología social (Munné, 1989). Pero esta disciplina hizo un gran esfuerzo para demostrar la importancia de la situación en la explicación del comportamiento humano. Tal esfuerzo se plasmó en notables investigaciones que resaltaban el papel determinante de la situación como factor explicativo de la conducta humana (Smith y Haslam, 2012) y que reducía y eliminaba la importancia de las variables de personalidad y variables cognitivas de los individuos como elementos explicativos de la conducta humana. Así, aunque la psicología social se situaba mayoritariamente alejada de las tesis conductistas, resaltó el determinismo situacional, asumiendo la idea de que las situaciones son determinantes de primer orden de la conducta social, coincidiendo así con los postulados conductistas. Esta idea, la del determinismo situacional, ya apareció en el psicólogo social conductista F. H. Allport (1924) que, aun rechazando los planteamientos más radicales del conductismo, sostenía que el estudio del impacto de la situación era más importante que el estudio de la conciencia (a la que consideraba tan sólo un epifenómeno). La influencia del poder de la situación quedó reflejada incluso en la definición que proporcionó G. Allport (1954) de la psicología social al resaltar que lo característico de esta disciplina era el estudio de la influencia de la presencia real o imaginada de otros seres humanos en los pensamientos, sentimientos y conductas de los individuos.

La complicidad y la colaboración de buena parte de la población alemana con el nazismo y el Holocausto durante la II Guerra Mundial proporcionó la base que inspiró valiosas investigaciones psicosociales en el marco de la psicología de los grupos, que resaltaron el postulado situacionista para explicar la crueldad humana. Estudios llevados a cabo por Milgram (1963; 1974) y por Zimbardo (Haney, Banks y Zimbardo, 1973; Zimbardo, 2004; 2008) situaron al poder de la situación como el factor explicativo de los comportamientos agresivos en ambientes totalitarios y justificativos de la tiranía, constituyendo la base empírica que la psicología social proporcionaba al concepto de la banalidad del mal (Arendt, 1963). Desde esta perspectiva, el mal triunfa debido a que personas decentes y ordinarias se convierten en individuos crueles cuando sus juicios son subvertidos por deferencia a grupos poderosos (Haslam y Reicher, 2007). En tales estudios se refutaba la importancia de las variables de personalidad en la explicación de los prejuicios hacia el exogrupo (a diferencia de como lo hicieron, por ejemplo, Adorno, Frenkel-Brunswik, Levinson y Sanford, 1950) y se resaltaba que los datos obtenidos demostraban que el poder de la situación suprimía las iniciativas y habilidades individuales, negando la posibilidad de cualquier comportamiento regido por la agencia. Entendiendo por ésta la capacidad y la intención por parte de los individuos de controlar su propia conducta y sus resultados (Bandura, 2006). El resumen de los resultados de estas investigaciones no refleja la elevada variabilidad comportamental que se obtenía entre los participantes y no apreciaba que las personas ejercían agencia evaluando la situación y determinando el mejor modo de seguir siendo fiel a sus convicciones y creencias (Swann y Jetten, 2017). Si se vuelve a visitar tales estudios, observando con más detalle los resultados obtenidos, se puede descubrir el papel desempeñado por la agencia, lo que implica un desafió a las explicaciones proporcionadas por los propios autores y exige la necesidad de llevar a cabo una reformulación teórica que supere la visión clásica dominada por una visión negativa de la realidad grupal y su influencia en el ser humano (Canto, 2019). El consenso que ha existido en el apoyo del concepto de la banalidad del mal está siendo seriamente cuestionado tanto por la revisión de los principales estudios psicosociales que le proporcionaban apoyo empírico (Milgram, 1974; Zimbardo, 2008), como por parte de historiadores centrados en el tema del Holocausto y del nazismo (Cesarani, 2004; Koonz, 2003; Lozowick, 2002; Rees, 1997; Vetlesen, 2005).

\section{Cuestionamiento histórico al concepto de la banalidad del mal}

El concepto de la banalidad de mal fue desarrollado por la filósofa Hannah Arendt (1963) tras asistir al juicio contra Adolf Eichmann, celebrado en Jerusalén. El 11 de abril de 1961 se inició el juicio contra Eichmann, jefe del subdepartamento de la oficina de seguridad del Tercer Reich y máximo responsable por parte de los nazis del plan para exterminar a los judíos. El alto mando nazi cuidó la imagen que dio de sí mismo en el juicio. Los asistentes no vieron en primer lugar a una persona sádica que había cometido crímenes monstruosos. Vieron a un hombre mayor, bastante gris, con apariencia de ser un simple funcionario. En la sala del juicio se encontraba Hannah Arendt como reportera de un periódico 
norteamericano. Ella, cuando escribió sobre el juicio, dijo que vio a un hombre sumamente obediente, que se sentía orgulloso del trabajo bien hecho, aunque su trabajo implicó exterminar a millones de personas. Arendt no quiso decir con la expresión la banalidad del mal que los actos de exterminio cometidos por los nazis fueran banales, sino que los motivos que los sustentaban eran banales, ya que los individuos estaban más centrados en realizar adecuadamente la tarea y agradar a sus superiores que en las consecuencias de sus actos. Esto explicaría que personas ordinarias pudieran cometer actos extremadamente malvados sin una motivación especial, sin características psicológicas distintas a las de la mayoría. Este concepto recibió supuestamente apoyo empírico por parte de la investigación de Milgram (1974) sobre la obediencia. El propio Milgram se inspiró en el concepto de banalidad del mal de Arendt (1963) para explicar los niveles de obediencia obtenidos en sus investigaciones. El estudio de Arendt sobre Eichmann y el Holocausto proporcionó la relevancia social a los estudios de Milgram y éstos proporcionaron credibilidad científica a las aportaciones de aquélla. Según Milgram, habría demostrado lo que Arendt había observado en el juicio contra el líder nazi: las personas ordinarias pueden infligir daños extraordinarios a otros seres humanos, por el simple hecho de prestar atención a las instrucciones de la autoridad y a la tarea y no a sus consecuencias (Canto y Álvaro, 2015).

La perspectiva de la banalidad del mal sigue siendo muy influyente hasta nuestros días, pero cuestionada por importantes críticas (Haslam y Reicher, 2007). Varios historiadores especialistas en el tema del Holocausto y la Alemania nazi han considerado el rol de la agencia moral en los actos de genocidio, prestándose una especial atención al caso de Eichmann y sus colaboradores (Cesarani, 2004; Koonz, 2003; Lozowick, 2002; Rees, 1999; Vetlesen, 2005). Cesarani (2004) argumentó que Arendt sólo estuvo los primeros cinco días del juicio contra Eichmann, en los que éste proporcionó una imagen muy elaborada de sí mismo buscando la exculpación. Se presentó como un burócrata obediente y no como un fanático nazi. Arendt no estuvo presente en los días del juicio en el que un número elevado de testigos mostraron una visión muy distinta de Eichmann. Se llegó a demostrar que era un nazi convencido y que su posición respecto a los judíos fue cambiando a medida que se identificaba más con el nazismo y ascendía en el escalafón del partido nazi. Si bien, en un principio, defendía que los judíos debían ser expulsados de Europa, pasó a defender con posterioridad el empleo de los campos de exterminio como método para conseguir la solución final. Eichmann no fue sólo un oficial nazi que siguió órdenes de sus superiores, sino que fue muy creativo e innovador buscando medidas más eficaces para exterminar a los judíos (Rees, 1999). Como ha documentado Cesarani (2004), Eichmann mostró una gran eficacia exterminando a los judíos húngaros en 1944 con métodos muy originales, entrando incluso en discusiones con Heinrich Himmler (su superior) sobre los métodos más adecuados y eficaces que debían ser empleados. Eichmann era consciente de las muertes que conllevaba sus decisiones y era consciente del rechazo que provocaba sus actos en otras personas alejadas del nazismo. Nunca mostró remordimiento ni arrepentimiento, incluso después de finalizar la II Guerra Mundial. Cesarani (2004) concluyó que Eichmann eligió ser un genocida. Vetlesen (2005), en esta misma línea, afirmó que los nazis sabían lo que estaban haciendo y creían en lo que hacían. Para Vetlesen, los crímenes cometidos no fueron resultado de la banalidad del mal, sino de individuos socializados en esa ideología genocida. Cesarani (2004) ha sostenido que la perspectiva sustentada por el concepto de la banalidad del mal ha servido para entorpecer un adecuado entendimiento de lo que aconteció durante el Holocausto.

\section{Evidencia empírica del concepto de la banalidad del mal: Milgram y Zimbardo}

Una de las investigaciones que se presentó en la literatura psicosocial como un claro ejemplo de apoyo empírico al concepto de la banalidad del mal (Arendt, 1963), lo constituyó el conjunto de experimentos realizados por Milgram (1963; 1974) en su análisis del proceso de obediencia. Milgram (1963) diseñó una situación experimental en la que cuarenta participantes (varones, con edades comprendidas entre los 20 y 50 años) creyeron que estaban colaborando en una investigación sobre aprendizaje. Cuando un sujeto experimental llegaba al laboratorio de la Universidad de Yale, se encontraba con un hombre de edad madura que declaraba que él también participaba en la experiencia. El experimentador les explicaba que la investigación trataba sobre la influencia del castigo en el aprendizaje. Tras un sorteo amañado, se le asignaba al sujeto experimental el rol de profesor y al otro hombre (que era un cómplice del experimentador) se le asignaba el rol de alumno. El experimentador informaba al profesor (sujeto experimental) que su tarea consistiría en leer pares de palabras al "alumno", verificar si era capaz de repetirlas e infringirle una descarga eléctrica por cada fallo. Para aplicar las descargas, se disponía de un supuesto generador con 30 interruptores (de 15 en 15 voltios) que llegaba a los 450 voltios. Durante el experimento, en el cual el castigo infringido era simulado, el "alumno" cometía numerosos errores y el "profesor" recibía instrucciones por parte del experimentador de que debía ir aumentando 
progresivamente el nivel de descargas. Tanto el "alumno" como el experimentador seguían un guion preestablecido. El "alumno" comenzaba a equivocarse y el experimentador daba instrucciones al "maestro" para que aplicara las descargas eléctricas pertinentes. Al llegar a 75 voltios, el "alumno" reflejaba dolor. A los 150 voltios, solicitaba dejar el experimento. A los 300 voltios, rehusaba seguir respondiendo y seguía solicitando abandonar el estudio. A los 330 voltios, dejaba de responder. El experimento que constituyó la línea base (Milgram, 1974), obedecieron hasta los 450 voltios el $65 \%$ de la muestra experimental: 26 participantes obedecieron y 14 desobedecieron. Milgram no disponía de un modelo teórico cuando inició su programa de investigación sobre la obediencia en la década de los 60 del siglo pasado, llevando a cabo treinta variaciones de sus estudios y obteniendo niveles de obediencia que oscilaban entre el 0 y el $100 \%$. Fue al publicar su libro en 1974 cuando proporcionó una teoría que diferenciaba dos estados psicológicos para explicar por qué los individuos obedecían en sus experimentos: a) el estado de autonomía, en el cual las personas se sienten responsables de sus actos y utilizan su propia conciencia como guía de comportamiento y b) el estado agéntico, en el cual las personas que se encuentran en este estado consideran que forman parte de una estructura jerárquica y sostienen que las autoridades son las responsables de sus actos y utilizan las órdenes de las autoridades como guía de acción correcta. Este estado sería el que rige y determina el nivel de obediencia en los experimentos diseñados por Milgram (1974).

Sin embargo, el poder de la situación en los experimentos realizados por Milgram (1974) estuvo lejos de ser absoluto (Canto y Álvaro, 2015; Haslam y Reicher, 2017; Swann y Jetten, 2017). Muchos participantes desobedecieron y muchos reflejaron una fuerte tensión a lo largo de experimento, aun cuando Milgram diseñó la situación experimental con extremo cuidado para provocar que los sujetos fuesen obedientes hasta el final (Russell, 2011). Aun cuando la presión de la fuente de autoridad era elevada, muchos participantes reflejaban un grado considerable de conexión con el "aprendiz" en detrimento de la conexión que podían mostrar con el experimentador. En concreto, si aumentaba la conexión con el experimentador, se incrementaba la complacencia con él, pero si aumentaba la conexión con el "aprendiz", decrecía el nivel de obediencia (Haslam y Reicher, 2012). Como sostienen Swann y Jetten (2017), la obediencia que obtuvo Milgram reflejaba la capacidad de los individuos de actuar conectados con otros individuos más que la capitulación a las demandas de una autoridad. El apoyo a esta tesis se obtiene tras el análisis de las instrucciones proporcionadas por el experimentador cuando los participantes se negaban a seguir sus órdenes (Burger, Girgis y Manning, 2011). El experimentador comenzaba de una forma suave (1 "Por favor, continúe") e incrementaba la intensidad a medida que el participante desestimara obedecer. Cuando el experimentador debía recurrir a las instrucciones de mayor intensidad (4a "Usted no tiene otra opción, usted debe continuar"), el nivel de obediencia disminuía significativamente (Haslam, Reicher y Birney, 2014). Estos datos hacen más inteligible el motivo por el cual Milgram indicó en una nota hallada en sus archivos si no era más preciso denominar al fenómeno que estaba estudiando como "un fenómeno de cooperación" y no como un "un fenómeno de obediencia" (Haslam, Reicher, Millard y McDonald, 2015).

El papel de las relaciones que se establecen entre las personas que se enfrentan a una autoridad es un factor de suma importancia para comprender los niveles de obediencia obtenidos (Swann y Jetten, 2017). Basándose en el paradigma de obediencia diseñado por Milgram (1963), Gamson, Fireman y Rytina (1982) dieron la oportunidad a los participantes de su experimento de formar coaliciones para enfrentarse a las órdenes de la autoridad. Los resultados obtenidos mostraron que los niveles de rebeldía fueron bastante elevados y la obediencia bastante baja. La mitad de los grupos rehusaron seguir las órdenes proporcionadas por la autoridad y sólo obedecieron el $12 \%$ de los participantes. Estos datos nos obligan a reconsiderar la postura del propio Milgram (1974) que sostuvo que las personas obedecen de forma rutinaria cediendo a la presión de la situación de forma acrítica. De hecho, tal como demostraron Gamson et al. (1982), el tipo de coaliciones que formaron las personas fue un papel determinante para comprender cómo se enfrentaban a las órdenes emitidas por una autoridad.

El experimento de la prisión de Stanford (EPS; Haney et al., 1973), llevado a cabo a lo largo del verano de 1971 en la Universidad de Stanford, ha sido considerado como una de las investigaciones más destacadas de la psicología social y se ha presentado como muestra de apoyo empírico al concepto de la banalidad del mal. El interés que suscitó fue más allá de la psicología social e influyó a otras ciencias sociales. Como nos recuerdan Haslam, Reicher y Van Bavel (2019), el EPS se encuentra referenciado en la inmensa mayoría de los libros de texto de la disciplina (Griggs, 2014). Constituye un elemento clave en los medios de comunicación para explicar la tiranía y la opresión, proporcionando un material disponible en Internet muy influyente (www.prisionexp.org). Además ha inspirado un libro muy famoso sobre el mal (Zimbardo, 2008) y varias películas sobre el tema. Todo ello ha conducido a que el EPS sea considerado por millones de personas la pieza clave para comprender la naturaleza de la crueldad humana (Haslam et al., 2019). 
EI EPS llegó a ser famoso por demostrar cómo jóvenes estudiantes universitarios de la prestigiosa Universidad de Stanford, ajustados desde el punto de vista psicológico, libres de cualquier psicopatología, dañaron cruelmente a ciudadanos inocentes simplemente como consecuencia de haber sido asignados a un rol de guardia en un contexto carcelario simulado, mientras que los que fueron asignados al rol de preso asumieron un comportamiento sumiso. Zimbardo y su equipo afirmaron que el mero hecho de ponerse el uniforme en un contexto carcelario (ya como guardia, ya como preso), era suficiente para transformar a los participantes en guardias agresivos y crueles y a los presos en personas dóciles y sumisas. Esta transformación se producía automáticamente y sin ningún tipo de instrucciones por parte de los investigadores. Así, una investigación que fue diseñada para que durara catorce días, tuvo que ser interrumpida al final de la primera semana, motivada por la extrema crueldad mostrada por los guardias y por la indefensión mostrada por los presos. Estos, tras un intento frustrado de motín, se derrumbaron psicológicamente y cayeron en la apatía más profunda. Incluso, los investigadores reconocieron públicamente que acabaron comportándose como jefes de prisión y trabajaron más horas para garantizar la seguridad y el orden en la cárcel simulada en los sótanos de su universidad.

En la misma década en la que el EPS tuvo lugar, ya recibió importantes críticas (Fromm, 1973; Banuazizi y Movahedi, 1975; Levibond, Mithiran y Adams, 1979, etc.). Actualmente, el análisis más detallado de los resultados y de los archivos disponibles del EPS ha propiciado nuevas evidencias que han inspirado la formulación de serias críticas a las conclusiones formuladas por Zimbardo (Griggs, 2014; Haslam y Reicher, 2007; 2012; Haslam et. al., 2019; Le Texier, 2018; 2019; Swann y Jetten, 2017). Muchos participantes no se conformaron al rol que les fue asignado en la situación experimental. La complacencia con la que se asumió los roles no fue algo universal. Muchos prisioneros se resistieron a la autoridad de la prisión hasta el final (Le Texier, 2018; 2019). De hecho, en la primera fase del estudio, los prisioneros desafiaron a los guardias. Cuando estos respondieron castigando la resistencia mostrada por los presos, algunos de ellos aumentaron sus conductas de insubordinación encaramándose en una de las celdas, defendiéndola con una barricada. Los guardias abortaron la rebelión utilizando la fuerza, pero Zimbardo nunca explicó cómo fue el proceso de toma de decisión por el cual los guardias eligieron llevar a cabo esas conductas que mostraron en la cárcel simulada para acabar con el motín de algunos presos (Swann y Jetten, 2017). Zimbardo (2008) sí intervino para evitar futuros intentos de rebelión por parte de los prisioneros. Ofreció a uno de los presos un tratamiento preferencial a cambio de que actuara como "un chivato" en el seno de la prisión. Este nuevo preso hizo ver al resto que era imposible salir de la misma, lo que produjo un gran impacto sobre ellos: dejaron de actuar como un colectivo y los guardias ya tuvieron muy pocos problemas para aplastar la resistencia de los presos que actuaban solos y sin coordinación grupal.

Entre los guardias hubo una elevada variabilidad de comportamientos (Haslam y Reicher, 2012; Haslam et al., 2019). Un tercio de los mismos llegó a comportarse de forma tiránica en su uso arbitrario del poder. De los dos tercios restantes, la mitad mostró un comportamiento duro pero justo con los presos y la otra mitad fue afable. Por tanto, muchos guardias modularon sus respuestas al asumir el rol que les asignaron. La variabilidad de comportamiento observada tanto en guardias como en prisioneros exige una explicación más precisa que la proporcionada por Zimbardo (2008), ya que la asignación de roles no puede ser considerada como suficiente para provocar las conductas observadas. No hay dudas de que algunos de los participantes con el rol de guardias asumieron un comportamiento punitivo y tiránico. Pero junto con la asignación de roles, Zimbardo y su equipo proporcionaron instrucciones acerca del comportamiento que debían seguir los guardias (Le Texier, 2019).

La existencia de evidencia indirecta nos sugiere que las instrucciones proporcionadas a los guardias por parte de los investigadores fueron determinantes en los resultados del estudio de Zimbardo. Concretamente, en el año 2002, Alex Haslam y Stephen Reicher realizaron un estudio de campo, en colaboración con la BBC (televisión pública británica), que se conoce con el nombre del estudio de la prisión de la BBC, un estudio que se puede comparar con el realizado en la Universidad de Stanford treinta años atrás (Reicher y Haslam, 2006). Estos investigadores no tuvieron intención de replicar el experimento de la prisión de Zimbardo. El estudio de la prisión de la BBC es definido por Haslam y Reicher como un estudio sobre la conducta de personas que ocupan posiciones dominantes y subordinadas y de las relaciones que se establecen entre ellas. Participaron quince personas, todos varones y psicológicamente bien adaptados. Se dividieron aleatoriamente en dos grupos: un grupo de guardias (cinco personas; estatus alto) y un grupo de prisioneros (diez personas; estatus bajo). El estudio duró ocho días. Los guardias tenían el control de la prisión y se identificaron con su grupo desde el primer momento. Los prisioneros se identificaron con su grupo y desafiaron a los guardias cuando observaron que no era posible mejorar su posición a través del esfuerzo individual. En este estudio, los guardias no llegaron a ser autoritarios ni los prisioneros sumisos. Aunque el estudio de la prisión de la BBC se 
diferenciaba en numerosos aspectos del EPS, se mostró que la asignación de roles no produjo las conductas observadas por Zimbardo y su equipo. Swann y Jetten (2017) sostienen que se debe prestar una especial atención a la posibilidad de formación de coaliciones en ambos estudios. En el EPS, los investigadores formaron coaliciones con los guardias y les animaron a adoptar conductas autoritarias contra los presos, los cuales se desanimaron a formar coaliciones una vez transcurridos los primeros días del estudio. En el estudio de Reicher y Haslam (2006), los investigadores no ofrecieron ningún tipo de instrucciones a los participantes. Aun así, los guardias decidieron formar coaliciones. Siendo idéntica la situación en ambos estudios al asignarles a los participantes distintos roles al azar en un contexto carcelario simulado, se diferenciaban en el rol asumido por los investigadores y en el tipo de conductas mostradas por los participantes.

Así, pues, por una parte, la revisión llevada a cabo por historiadores sobre la realidad del Holocausto ha resaltado un nuevo enfoque que se aleja del concepto de la banalidad del mal. Por otra parte, la teoría formulada por Milgram (1974) y la proporcionada por Zimbardo (2008) no pueden ser consideradas explicaciones plausibles de los resultados obtenidos. Pero siendo así, en estas investigaciones se pudo observar cómo muchos de los participantes optaron por conductas que implicaban dañar y abusar de otras personas. Ello exige aplicar a estos resultados otros planteamientos teóricos que puedan explicar la elevada variabilidad de las conductas observadas en ambos contextos experimentales. La línea de investigación llevada a cabo por Haslam y Reicher (Haslam y Reicher, 2012; Haslam et al., 2019; Reicher, Haslam y Hopkins, 2005) ha resaltado el papel que desempeñaron las relaciones de liderazgo de identidad en el transcurso de las dinámicas grupales que acontecieron en los experimentos de Milgram y Zimbardo.

\section{El papel de la identidad social en las relaciones de liderazgo: cuando el líder "(re)crea" una nueva identidad social}

Según Haslam y Reicher (2012; 2017; Haslam et al., 2019), los resultados de los experimentos de Milgram (1974) y del EPS (Zimbardo, 2008) pueden ser explicados por el proceso del liderazgo de identidad. Estos autores se basan en la perspectiva de la identidad social, que incluye tanto la teoría de la identidad social (Tajel y Turner, 1979) como el posterior desarrollo de la teoría de la categorización del yo (Turner, Hogg, Oakes, Reicher y Wetherell, 1987). Para los teóricos de la identidad social, los individuos se definen a sí mismos no sólo como individuos únicos sino también como miembros pertenecientes a grupos (Turner et al., 1987). La identidad social de un individuo está formada por las identidades que se derivan de la pertenencia a distintos grupos sociales. Como un individuo puede pertenecer a varios grupos, éste posee diversas identidades sociales, que se ponen de manifiesto en diversos contextos. De hecho, la identificación social compartida es la que posibilita la formación del grupo y la acción colectiva (Turner, 1981). Cuando un individuo se percibe a sí mismo como perteneciente a cualquiera de los grupos de los que forma parte, tiende a verse a sí mismo y a los demás en función de las categorías a las que pertenece. Una vez que se autocategoriza en términos colectivos, se percibe y se evalúa en términos del grupo social (Turner et al., 1987). Si el grupo es definido positivamente y es fuente de orgullo, aumenta la probabilidad de identificación con él, ya que tiene repercusiones en su autoestima (Tajfel y Turner, 1979).

Cuando los individuos comparten la misma identidad social al pertenecer al mismo grupo, se transforma la relación entre las personas del endogrupo del tal modo que permite la acción colectiva coordinada, aumentando su nivel de confianza y el grado de cooperación entre ellas (Tyler y Blader, 2000). Además, cuando se comparte una identidad social (con sus valores, metas, etc.), es probable que uno o más miembros del grupo representen lo que tienen en común, con lo que la identidad social compartida hace posible la emergencia del liderazgo (Reicher, Haslam y Hopkins, 2005). El concepto clave para explicar la influencia desde esta teoría es el concepto de influencia informativa referencial, que trata de la influencia que se produce entre individuos que pertenecen a una misma categoría social. Según Turner (1991), tras el proceso de la categorización del yo se hace saliente la categoría social pertinente y el individuo conoce las normas estereotipadas de la categoría y se las asigna, asumiendo una serie de conductas y opiniones que se derivan de aquélla. Es decir, cuando las personas adoptan una identidad social, se pone en marcha un proceso de autoestereotipia. Esto implica que procuran conocer las normas, los valores y el contenido que caracterizan a dicho grupo, conformándose al mismo. Se aceptan las opiniones y las actitudes del grupo porque definen a los individuos y establecen lo que es importante para ellos en cuanto miembros de ese grupo (Reicher, Haslam, Spears y Reynolds, 2012). Quienes se identifican con una categoría social son influidos por la información relativa a la identidad del grupo. A su vez, quienes están en una posición de poder dar una definición autorizada de la identidad del grupo (es decir, aquellos que son percibidos como prototípicos), están en condicio- 
nes de ejercer influencia en el endogrupo al ser percibidos como líderes (Haslam, Reicher y Platow, 2013).

Los líderes ejercen el liderazgo en un grupo, afectando a los seguidores, debido a que comparten la misma identidad social. Esta permite a los individuos definirse como "nosotros". La definición de quienes están incluidos en esa categoría social determina el tamaño del grupo; la definición de lo que significa ser un miembro de la categoría (contenido de la categoría) determina la acción y el comportamiento del grupo y, por último, la definición de quién ejemplifica mejor la categoría (prototipo de la categoría) determina el liderazgo (Reicher y Hopkins, 2001). Cuando un individuo se define a sí mismo desde la identidad social, precisa tanto conocer el significado asociado a la categoría social como las implicaciones que conlleva. Gracias a la identidad social internalizada por los individuos del grupo, se estructuran y organizan la percepción social y la interacción social de tal modo que las pautas idiosincráticas de las personas se transforman en creencias consensuadas (Haslam et al., 2013). La identidad social compartida es la base del poder social colectivo (Turner, 2005). El hecho de que a un miembro del grupo se le considere representante de una identidad social es también una fuente importante de poder potencial. De hecho, la prioridad fundamental de los que quieren ser líderes es que se les consideren parte del grupo y portavoces del mismo. Pero, además, el líder necesita ser considerado un buen representante del grupo que lucha por los intereses colectivos, ejemplificando lo que les hace diferente de los exogrupos relevantes en el contexto comparativo donde opera (Turner y Haslam, 2001). Pero además los líderes para ser efectivos deben ser "emprendedores" de identidad social, ya que deben volver a definir quiénes son y deben hacer que los seguidores perciban sus ideas como la encarnación de quiénes son y lo que quieren llegar a ser (Haslam et al., 2013). Es decir, deben presentar sus propios mensajes y sus proyectos como una actualización de las normas y valores de las creencias del grupo, así como tener la capacidad de promover acciones que ayuden a sus grupos a conseguir sus objetivos (Haslam et al., 2019).

\section{Los resultados de los experimentos de Milgram sobre obediencia y del EPS desde la perspecti- va del liderazgo de identidad}

Haslam y Reicher (Haslam et al., 2014; Haslam et al., 2015; Reicher, Haslam y Smith, 2012) han iniciado una línea de investigación con el propósito de demostrar que el grado de identificación entre los participantes con la figura de autoridad es crucial en los niveles de obediencia obtenidos por Milgram (1974). Tras el análisis y revisión de los archivos del propio Milgram, se ha podido constatar que realizó un gran esfuerzo para conseguir que los participantes se identificaran con los (supuestos) objetivos de la investigación y asumieran que el uso progresivo de castigos era un método efectivo para mejorar el aprendizaje. Los sujetos experimentales desearon seguir las instrucciones proporcionadas por el investigador en la medida en que se identificaban con él y con la comunidad científica que representaba (Haslam et al., 2014; Reicher et al., 2012). Al identificarse con el investigador, los participantes colaboraron con la tarea diseñada por científicos y comenzaron a formar parte de un nuevo grupo prestigioso para ellos. Investigaciones diseñadas por Haslam y Reicher han puesto de manifiesto que el poder de los estímulos situacionales dentro del paradigma de Milgram deriva precisamente de la capacidad de estos para producir algún grado de identificación entre los participantes con el experimentador y la empresa científica. Así, por ejemplo, Haslam et al. (2019) afirman que los participantes se identifican más con el experimentador en estas condiciones: a) cuando se encuentran en la misma habitación, b) cuando el estudio se desarrolla en una institución prestigiosa y c) cuando el experimentador requiere a los participantes que continúen y no cuando les ordena taxativamente que lo hagan.

Los resultados del EPS y la interpretación de los mismos fueron expuestos a la comunidad científica principalmente en el artículo de Haney et al. (1973) y en el libro de Zimbardo (2008) publicado posteriormente, sin olvidar el vídeo publicado sobre el tema (Zimbardo, 1989) y un capítulo publicado en 2004 (Zimbardo, 2004). La nueva evidencia proporcionada por los archivos disponibles sobre el EPS ha permitido un análisis de nuevos datos que han salido a la luz (Le Texier, 2018; 2019). Estos nuevos datos han permitido comprobar el elevado grado de liderazgo ejercido por Zimbardo y su equipo y cómo tal liderazgo fue capaz de incidir en la creación de una identidad compartida entre los experimentadores y los guardias (Haslam et al., 2019). Le Texier (2019) destacó cómo los investigadores proporcionaron instrucciones muy precisas a los guardias durante largas sesiones, indicándoles que la ejecución de conductas de dominación y despóticas eran necesarias para alcanzar las metas propuestas por el estudio, haciéndoles ver que ellos eran parte del proyecto de investigación y su participación era fundamental para que concluyera con éxito. Esas instrucciones no se limitaron a las sesiones previas al estudio, sino que continuaron una vez finalizado el mismo. Según Le Texier (2019), los archivos han proporcionado datos que reflejan que los guardias eran conscientes de lo que se esperaba de ellos 
para conseguir los objetivos de la investigación. En estas sesiones formativas, donde participaban los guardias y los investigadores, se configuraron las reglas que debían regir el funcionamiento de la prisión. Concretamente, Le Texier (2019) ha destacado que de las diecisiete reglas que se establecieron para el funcionamiento de la prisión, once de ellas fueron diseñadas en estas sesiones, mientras que el resto fueron en gran medida adaptaciones a las circunstancias especiales del EPS. Así, se puede concluir que el nivel de intervención de Zimbardo y su equipo fue superior al reflejado en los artículos y libros publicados sobre el experimento (Haslam et al., 2019). Los experimentadores desempeñaron un rol muy activo en el propósito de guiar las conductas de los guardias. Zimbardo y su equipo apelaron al sentido de identidad compartida, definiendo a la dureza comportamental como un atributo necesario de la identidad del endogrupo que incluía a guardias e investigadores y conseguir así los objetivos del estudio: demostrar la toxicidad del sistema penal norteamericano.

Haslam at al., (2019) especifican que el efecto de un liderazgo, que interviene en la conformación de una nueva identidad, no implica que tal identificación entre los miembros del grupo provoque siempre un consenso entre todos los integrantes y que se elimine la posibilidad de realizar comportamientos autónomos no dictados por el líder. Según Haslam y Reicher (2012), desde el modelo del liderazgo de identidad, el comportamiento autónomo y creativo desde ser concebido como el resultado de un seguimiento implicado por parte de los miembros del grupo y no como un caso de conformidad pasiva. Las personas no asumen automáticamente las identidades sociales que se les imponen. De hecho, se pueden resistir a las categorías sociales e identidades que se les proponen, si éstas están en contradicción con otras identidades importantes para ellas.

Las revisiones de las investigaciones de Milgram (1974) y el EPS (Haney et al., 1973) han puesto de manifiesto que pocas personas espontáneamente se identifican con roles que requieren actuar con elevadas cotas de crueldad. Ello se consigue previa acción premeditada de los líderes en la creación de una identidad común en la que la crueldad es percibida como necesaria para la protección y avance del endogrupo. Tal como sostienen Reicher, Hopkins, Levine y Rath (2005) y Reicher, Haslam y Rath (2008), cuando se ejerce la violencia en contextos intergrupales, la agresión ejercida hacia el exogrupo por parte del endogrupo se cataloga como "legítima defensa" por parte de los líderes para que sea aceptada por el grupo. En el proceso que conduce a ello se pueden distinguir tres elementos estrechamente relacionados. En primer lugar, hay que interpretar la identidad del endogrupo de tal modo que el exogrupo quede excluido. En segundo lugar, el exogrupo debe ser percibido como un problema o, peor aún, como una amenaza para el endogrupo. Y, en tercer lugar, el endogrupo debe autopercibirse como un grupo virtuoso. Así se justifica que la amenaza que implique el exogrupo pueda ser eliminada con acciones violentas por parte del endogrupo, ya que tales acciones son etiquetadas como actos de legítima defensa. Entender la violencia y la crueldad requiere un análisis del liderazgo, de sus técnicas de persuasión y de las estrategias utilizadas para conseguir que las conductas crueles y violentas contra el exogupo sean percibidas como una acción digna en defensa de la causa noble del endogrupo (Haslam et al., 2019).

\section{Conclusiones}

El situacionismo ha constituido uno de los pilares metateóricos más importantes de la psicología social (Álvaro y Garrido, 2003; Munné, 1989). Desde esta perspectiva se han realizado dos de los experimentos más influyentes de la psicología social (Canto, 2019). Así, los estudios de Milgram (1974) sobre obediencia y el estudio de Zimbardo sobre la influencia de los roles (Haney et al., 1973) son recogidos en la inmensa mayoría de los manuales de la disciplina y han tenido un gran impacto en otras ciencias sociales y en la sociedad en su conjunto. El relato común que agrupa a estas investigaciones resalta el elevado poder de la situación pero encubre una variabilidad de comportamientos producidos en ambos contextos experimentales, la cual matiza y desafía las elaboraciones teóricas desarrolladas por los propios autores para explicar los resultados obtenidos. Fue tal el interés de la psicología social en analizar los procesos de influencia social responsables del control social, resaltando la conformidad y la obediencia en detrimento del estudio de los procesos responsables del cambio social (Moscovici, 1981), que Milgram y Zimbardo focalizaron la atención en los resultados con un sesgo confirmatorio para verificar sus hipótesis planteadas desde el postulado metateórico del situacionismo, desestimando la amplitud y variabilidad de los mismos. Y como afirman Swann y Jetten (2017), si la psicología social opta por una perspectiva metateórica unidimensional puede provocar que los investigadores estén presos de "orejeras teóricas" que malinterpreten los fenómenos psicosociales que estén investigando. La relectura de los estudios de Milgram y Zimbardo nos muestra cómo, ante situaciones muy influyentes, los participantes no abandonan estrategias de comportamiento imbuidas de algún grado de agencia. Aceptar la influencia de la situación (incluso en situaciones con mucho poder) no debe implicar recha- 
zar la posibilidad de comportamientos agénticos. Las personas ejercen agencia evaluando la situación y determinando el mejor modo de alcanzar sus metas mientras pertenecen fiel a sus creencias y disposiciones (Bandura, 2006). La conducta se da en una interacción de factores personales y situacionales, incluyendo la capacidad que tienen las personas de alterar el curso de la acción ante tales situaciones. Ejercer la agencia requiere ser receptivos a la situación y ser conscientes de las respuestas que se elaboran ante tales situaciones; por ello las presiones situacionales pueden incrementar o disminuir las expresiones de comportamientos agénticos. Según estos autores, la psicología social no debe tan sólo explicitar los mecanismos que tienen las situaciones para influir en el comportamiento humano, sino también descubrir y enumerar los sentimientos de agencia de las personas que son víctimas de condiciones sociales negativas que provocan sufrimiento, con la intención de capacitar a estas personas para superar sus condiciones sociales negativas. Si la psicología social se desprende de la ligazón situacionista, se amplía el número de fenómenos de su ámbito de estudio en los que se constata cómo el ser humano emprende distintos modos y distintos tipos de lucha para afirmarse a sí mismo.

Los estudios de Milgram (1974) sobre obediencia social muestran un buen conjunto de datos empíricos en los que se observan distintos niveles de influencia junto con una escasa e imprecisa elaboración teórica. Para Milgram sus investigaciones mostraban la tendencia del ser humano a obedecer de forma acrítica a la autoridad, acompañadas de un modelo explicativo que resaltaba el enorme poder de la situación en la conducta, en detrimento de las disposiciones personales. Para Blass (2004), Milgram tuvo la capacidad de identificar una de las pautas universales de la conducta social: los seres humanos tienen la propensión natural de obedecer a la autoridad. Sin embargo, el reanálisis de los datos obtenidos ha llevado al cuestionamiento tanto de las conclusiones de Milgram como de su modelo teórico (Reicher y Haslam, 2012; Swann y Jetten, 2019). Milgram nos proporcionó una situación experimental diseñada con todo detalle para provocar obediencia (Russell, 2011), pero no nos proporcionó igualmente una explicación satisfactoria. La noción de estado agéntico (Milgram, 1974) sostiene que, ante una autoridad, las personas pierden de vista sus valores y normas y ceden la responsabilidad de sus actos a la autoridad, preocupándose tan sólo de seguir las instrucciones que proceden de la autoridad y no de las consecuencias de sus actos de obediencia. Pero como afirman Reicher y Haslam (2012), no hay evidencia empírica de que las personas entren en ese estado agéntico, como tampoco hay evidencia empírica de que las variaciones en ese estado puedan explicar los distintos niveles de obediencia obtenidas con el paradigma experimental. Ni los resultados mostrados por Milgram (1974) ni el análisis histórico proporcionado por Arendt (1963) son válidos para poder ser utilizados como apoyos al concepto de la banalidad del mal ni el concepto del estado agéntico.

El estudio de Zimbardo (Haney et. al., 1973), a semejanza del trabajo de Milgram (1974), fundamentó empíricamente el concepto de la banalidad del mal. Según Zimbardo, se demostró que el descenso a comportamientos crueles y tiránicos viene determinado por una "tendencia natural" que tienen los individuos a conformarse automáticamente a los roles, sin que haya la necesidad de la existencia de una autoridad que empuje a tales comportamientos. Las personas que acceden son obligadas a realizar un rol que tiene algún grado de poder sobre otros individuos y abandonan cualquier elemento de su identidad que pudiera oponerse a las exigencias demandadas por ese rol y sucumben al mismo. Pero el reanálisis de los datos obtenidos en el EPS no confirma la teoría que Zimbardo empleó para explicar tales datos y pone en evidencia una elevada variabilidad comportamental entre los guardias y los prisioneros. Desde la óptica del liderazgo de identidad (Haslam et al., 2019), los guardias fueron objeto de acciones de liderazgo por parte de los investigadores. Estos se comprometieron con un liderazgo de identidad en un esfuerzo de fomentar la crueldad de los guardias, formando una identidad común entre ambos. Los experimentadores consiguieron que los guardias se identificaran con la causa del grupo, hicieron que las metas grupales fuesen percibidas como nobles y virtuosas $y$, por último, hicieron ver que dañar a otros era absolutamente necesario para alcanzar las metas del grupo. Los guardias, por su parte, como seguidores del proceso de liderazgo, asumieron la identidad común compartida con el líder, entendieron las acciones necesarias para ayudar a conseguir las metas y colaboraron respondiendo con un cierto entusiasmo y con acciones creativas y no sólo siguiendo literalmente las instrucciones de los investigadores. Por tanto, en el EPS se puede observar la intervención de líderes con el propósito de influir a los participantes del experimento en su rol de guardias, como del mismo modo en los experimentos de Milgram sobre obediencia la autoridad intervenía con el propósito de provocar la percepción de una identidad común entre los sujetos experimentales y la comunidad científica que representaba el propio experimentador (Haslam y Reicher, 2017; Russell, 2011).

Los estudios de Milgram y Zimbardo nos han mostrado que para comprender la ejecución de conductas crueles en ambientes grupales es necesario analizar el proceso de liderazgo en la creación de identidades sociales que normalicen la agresión hacia el exogrupo. Si bien Milgram y Zimbardo 
nos ayudaron a superar el reduccionismo psicologicista, según el cual la agresión y violencia grupal eran atribuidas exclusivamente a causas psicopatológicas individuales, ahora se debe superar otro reduccionismo que surge de sus propias investigaciones: aquel que atribuye la violencia grupal a la psicopatología de la situación, olvidando el papel que desempeñan los líderes en toda la dinámica grupal (Haslam y Reicher, 2007). Las personas no siguen a los líderes brutales ni ejecutan roles brutales en grupo de forma automática sin cuestionamiento. Ello exige una labor muy precisa por parte de los líderes para crear una identidad social común con los seguidores, en la que las acciones brutales hacia otras personas sean justificadas como actos de legítima defensa contra la amenaza que constituye el exogrupo.

La teoría del liderazgo de identidad surge desde la perspectiva de la identidad social (Haslam et al., 2011). Un líder de identidad opera desde una categoría social, (re)define lo que significa ser miembro de esa categoría, establece los límites con los exogrupos, el contenido específico que se atribuye a esos límites e interpreta las relaciones entre las categorías sociales (Reicher et al., 2005). Los líderes tóxicos que ejercen ese rol en organizaciones tiránicas justifican esas interpretaciones alejadas de un discurso democrático (abierto al debate y a posibles alternativas). Por el contrario, lo hacen utilizando un discurso autocrático, que da por sentada la interpretación expuesta por el líder y excluye la posibilidad de cualquier debate que le cuestione. Aquí se constata la existencia de dos tipos de liderazgos opuestos al liderazgo democrático: a) un líder jerárquico que defiende su identidad social apelando al carácter esencialista de su planteamiento, determinado por su supuesto conocimiento histórico de lo que implica ser de ese grupo y b) un líder autocrático que se presenta como personificación de la categoría social, lo que implica que cualquier crítica recibida sea percibida como una crítica dirigida al grupo (situando a quien ejerce la crítica al margen, es decir, como exogrupo), haciendo desaparecer el espacio de la oposición e iniciando o consolidando el camino de la autocracia (Reicher et al., 2005). Cuando se analiza la historia de los líderes en los regímenes dictatoriales y genocidas de nuestra época, se puede observar cómo tienen un gran conocimiento de cómo usar la identidad social para proyectos violentos. Saben multiplicar sus adeptos, saben provocar su agresividad y saben cómo dirigirla hacia exogrupos concretos. Conocer estas dinámicas, conocer la forma en la que (re)construyen identidades sociales propiciadoras de exclusión y discriminación, conocer los discursos repletos de odio hacia los exogrupos, en definitiva conocer cómo los líderes tóxicos han construido sus herramientas psicosociales del poder, es una tarea a la que nos debemos dedicar los psicólogos sociales que queremos denunciar las conductas de dominación y queremos colaborar en prácticas que conduzcan a la convivencia y mejora de las relaciones entre los seres humanos.

\section{Referencias}

1. Adorno, T. W., Frenkel-Brunswik, E., Levinson, D. \& Sanford, N. (1950). The authoritarian personality. Nueva York: Harper.

2. $\quad$ Allport, F. H. (1924). Social psychology. Boston. Houghton \& Mifflin.

3. Allport, G. W. (1954). The nature of prejudice. Cambridge, MA: Perseus Books.

4. Álvaro, J. L. \& Garrido, A. (2003). Psicología social. Perspectivas psicológicas y sociológicas. Madrid: McGraw Hill.

5. Arendt, H. (1963). Eichmann in Jerusalem: A report on the banality of evil. Nueva York: Penguin.

6. Banuazizi, A. \& Movahedi, S. (1975). Interpersonal dynamics in a simulated prison: A methodological analysis. American Psychologist, 30, 152-160. https://doi.org/10.1037/h0076835

7. Bandura, A. (2006). Toward a psychology of human agency. Perspectives on Psychological Science, 1, 164-180. https://doi.org/10.1037/e416902005-796

8. Blass, T. (2004). The man who shocked the world: The life and legacy of Stanley Milgram. Nueva York: Basic Books.

9. Burger, J. M., Girgis, Z. M. \& Manning, C. M. (2011). In their own words: Explaining obedience to authority through an examination of participants' comments. Social Psychological \& Personality Science, 2, 460-466. https://doi.org/10.1177/1948550610397632

10. Canto, J. M. (2019). Psicología de los grupos. Málaga: Aljibe.

11. Canto, J. M. y Álvaro, J. L. (2015). Más allá de la obediencia: Reanálisis de la investigación de Milgram. Escritos de Psicología, 8, 13-20. https://doi.org/10.5231/psy.writ.2015.0701

12. Cesarani, D. (2004). Eichmann: His life and crimes. Londres: Heinemann.

13. Fromm, E. (1973). The anatomy of human destructiveness. Nueva York: Holt, Rinehart and Winston.

14. Gamson, W. B., Fireman, B. \& Rytina, S. (1982). Encounters with unjust authority. Hounwood, IL: Dorsey Press. 
15. Griggs, R. (2014). Coverage of the Stanford Prison Experiment in introductory psychology textbooks. Teaching of Psychology, 41, 195-203. https://doi.org/10.1177/0098628314537968

16. Haney, C., Banks, C. \& Zimbardo, P. (1973). Interpersonal dynamics in a simulated prison. International Journal of Criminology and Penology, 1, 69-97. https://doi.org/10.21236/ad0751041

17. Haslam, S. A. \& Reicher, S. (2007). Beyond the banality of evil: Three dynamics of an interactionist social psychology of tyranny. Personality and Social Psychology Bulletin, 33, 615-622. https:// doi.org/10.1177/0146167206298570

18. Haslam, S. A. \& Reicher, S. (2012). When prisoners take over the prison: A social psychology of resistance. Personality and Social Psychology Review, 16, 154-179. https://doi. org/10.1177/1088868311419864

19. Haslam, S. A. \& Reicher, S. (2017). 50 years of "obedience to authority": From blind obedience to engaged followership. Annual Review of Law and Social Science, 13, 59-78. https://doi. org/10.1146/annurev-lawsocsci-110316-113710

20. Haslam, S. A., Reicher, S. \& Birney, M. E. (2014). Nothing by mere authority: Evidence that in an experimental analogue of the Milgram paradigm participants are motivated not by orders but by appeals to science. Journal of Social Issues, 70, 473-488. https://doi.org/10.1111/josi.12072

21. Haslam, S. A., Reicher, S., Millard, K. \& McDonald, R. (2015). "Happy to have been service": The Yale archive as a window into the engaged followership of participants in Milgram's "obedience" experiments. British Journal of Social Psychology, 54, 55-83. https://doi.org/10.1111/bjso.12074

22. Haslam, S. A., Reicher, S. \& Platow, M. (2011). The new psychology of leaderships: Identity, influence and power. Nueva York: Psychology Press.

23. Haslam, S. A., Reicher, S., \& Van Bavel, J. J. (2019). Rethinking the nature of cruelty: The role of identity leadership in the Stanford Prison Experiment. American Psychologist, 74, 809-822. https://doi.org/10.31234/osf.io/b7crx

24. Koonz, K. (2003). The Nazi conscience. New Haven, CT: Harvard University Press.

25. Le Texier, T. (2018). Histoire d'un mensonge: Enquête sur l'experience de Stanford. Paris: Editions la Découverte.

26. Le Texier, T. (2019). Debunking the Stanford Prison Experiment. American Psychologist, 74, 823839. https://doi.org/10.31234/osf.io/mjhnp

27. Lewin, K. (1951). Field theory in social science. Nueva York: Harper.

28. Lovibon, S. H., Mithiran, X. \& Adams, W. G. (1979). The effects of three experimental prison environments on the behaviour of non-convict volunteer subjects. Australian Psychologist, 14, 273-287. https://doi.org/10.1080/00050067908254355

29. Lozowick, Y. (2002). Hitler's bureaucrats: The nazis security police and the banality of evil. Londres: Continuum.

30. Milgram S. (1963). Behavioral study of obedience. Journal of Abnormal and Social Psychology, 67, 371-378. https://doi.org/10.1037/h0040525

31. Milgram, S. (1974). Obedience to Authority: An experimental view. Nueva York: Harper and Row.

32. Moscovici, S. (1981). Psicología de las minorías activas. Madrid: Morata.

33. Munné, F. (1989). Entre el individuo y la sociedad. Marcos y teorías actuales sobre el comportamiento interpersonal. Barcelona: PPU.

34. Rees, L. (1997). The nazis: A warning from history. Londres: BBC Books.

35. Reicher, S. \& Haslam, S. A. (2006). Rethinking the psychology of tyranny: The BBC Prison Study. British Journal of Social Psychology, 45, 1-40. https://doi.org/10.1348/014466605x81720

36. Reicher, S. \& Haslam, S. A. (2012). Obedience. Revisiting Milgram's shock experiments. En J. R. Smith \& S. A. Haslam (Eds.), Social Psychology. Revisiting the classic studies (pp. 106-125). Londres: Sage.

37. Reicher, S., Haslam, S. A. \& Hopkins, N. (2005). Social identity and the dynamics of leadership: Leaders and followers as a collaborative agents in the transformation of social reality. Leadership Quartely, 16, 547-568. https://doi.org/10.1016/j.leaqua.2005.06.007

38. Reicher, S., Haslam, S. A., Spears, R. \& Reynolds, K. (2012). A social mind: The context of John Turner's work and its influence. European Review of Social Psychology, 23, 344-385. https://doi. org/10.1080/10463283.2012.745672

39. Reicher, S., Haslam, S A. \& Rath, R. (2008). Making a virtue of evil: A five-step social identity model of the development of collective hate. Social and Personality Psychology Compass, 2/3, 1313-1344. https://doi.org/10.1111/j.1751-9004.2008.00113.x 
40. Reicher, S., Haslam, S. A. \& Smith, J. R. (2012). Working towards the experimenter: reconceptualizing obedience within the Milgram paradigm as identification-based followership. Perspectives on Psychology Science, 7, 315-324. https://doi.org/10.1177/1745691612448482

41. Reicher, S. \& Hopkins, N. (2001). Self and nation. Londres: Sage.

42. Reicher, S., Hopkins, N., Levine, M., \& Rath, R. (2005). Entrepreneurs of hate and entrepreneurs of solidarity: Social identity as a basis for mass communication. International Review of Red Cross, 87, 621-637. https://doi.org/10.1017/s1816383100184462

43. Russell, N. J. (2011). Milgram's obedience to authority experiments: Origins and early evolution. British Journal of Social Psychology, 50, 140-162. https://doi.org/10.1348/014466610x492205

44. Smith, J. R. \& Haslam, S. A. (2012). Social psychology. Revisiting classic studies. Londres: Sage.

45. Swann, W. B. \& Jetten, J. (2019). Restoring agency to the human actor. Perspectives on Psychological Science, 12, 382-399. https://doi.org/10.1177/1745691616679464

46. Tajfel, H. \& Turner, J. C. (1979). An integrative theory of intergroup conflict. En W. G. Austin \& S. Worchel (Eds.), The social psychology of intergroup relations (pp. 33-77). Monterey, CA: Brooks/ Cole.

47. Turner, J. C. (1981). Towards a cognitive redefinition of the social group. Cahiers de Psychologie Cognitive, 1, 93-118.

48. Turner, J. C. (1991). Social influence. Buckingham: Open University Press.

49. Turner, J. C. (2005). Explaining the nature of power: A three-process theory. European Journal of Social Psychology, 35, 1-22. https://doi.org/10.1002/ejsp.244

50. Turner, J. C. \& Haslam, S. A. (2001). Social identity, organization and leadership. En M. E. Turner (Ed.), Groups at work: Advances in theory and research (pp. 25-65). Hillsdale, NJ: Erlbaum.

51. Turner, J.C., Hogg, M. A., Oakes, P. J., Reicher, S. \& Wetherell, M. S. (1987). Rediscovering the social group. A self-categorization theory. Oxford: Basil Blackwell.

52. Tyler, T. R. \& Blader, S. L. (2003). The group engagement model: Procedural justice, social identity, and cooperative behaviour. Personality and Social Psychology Review, 7, 349-361. https:// doi.org/10.1207/s15327957pspr0704 07

53. Vetlesen, A. J. (2005). Evil and human agency: Understanding collective evildoing. Cambridge: Cambridge University Press.

54. Zimbardo, P. (1989). Quiet rage: The Stanford prison study [video]. Stanford, CA: Stanford University.

55. Zimbardo, P. (2004). A situacionist perspective on the psychologist of evil: Understanding how good people are transformed into perpetrators. En A. Miller (Ed.), The social psychology of good and evil (pp. 21-50). Nueva York: Guilford.

56. Zimbardo, P. (2008). El efecto Lucifer. El porqué de la maldad. Barcelona: Paidós.

RECIBIDO: 3 de febrero de 2020 MODIFICADO: 3 de junio de 2020 ACEPTADO: 18 de julio de 2020 